\title{
Molecular Dynamics Simulations of Defect Production in Graphene by Carbon Irradiation
}

\author{
J. Martinez-Asencio, M. J. Caturla ${ }^{a}$ \\ Dept. Física Aplicada, Facultad de Ciencias, Fase II, Universidad de Alicante, Alicante E-036090,Spain
}

\begin{abstract}
We present molecular dynamics simulations with empirical potentials to study the type of defects produced when irradiating graphene with low energy carbon ions $(100 \mathrm{eV}$ and $200 \mathrm{eV})$ and different dose rates. Simulations show the formation of very stable structures such as dimers, single chains of carbons and double chains of carbons. These structures are similar to those described in the literature, observed experimentally when irradiating graphene. For high doses or dose rates, the formation of nanopores is observed, similar to previous results by other authors for higher energies of the implanted ions. These simulations show how tunning the different parameters of irradiation conditions can be used to selectively create defects in graphene.
\end{abstract}

Keywords: molecular dynamics; graphene; defects; irradiation

\section{Introduction}

Graphene is, undoubtedly, one of the most promising materials, with applications in many different fields [1], from energy storage [2] to microelectronics [3] or biological applications [4] among many others. In the field of radiation effects, graphene has a special interest due to the possible changes of electrical and mechanical properties of this system induced by defects [5] and the formation of very stable structures under some types of irradiation [6]. Modification of the graphene layer by electron [6, 7] or ion implantation [8] has increasing attention since it could be a way of controlling the production of defects. For these methods to be efficient and feasible it is important to understand the type of defects produced under the different irradiation conditions, such as irradiation type (electrons or ions), temperature, energy and dose.

In this work we use molecular dynamics simulations with empirical potentials to study the effect of low energy collisions by carbon atoms on a graphene sheet. Molecular dynamics simulations have been used extensively to study carbon nanostructures [9]. In graphene the main focus has been on studying vacancy and vacancy clusters due to their effect on the electrical properties of this material [5] as well as fracture and mechanical behaviour [10] due to its enhanced properties. But there is also an important number of publications related to irradiation of graphene under different conditions [7, 11-13]. In particular, irradiation of graphene with carbon atoms has been studied by Bellido and Seminario [11]. They performed simulations of individual implantation of single carbon ions with energies between $0.1 \mathrm{eV}$ and $100 \mathrm{keV}$ and observed the formation of single vacancies and double vacancies. Li et al [12] studied the continuous irradiation of graphene with different ions. Defects produced by electron irradiation have also been studied using molecular dynamics [13]. In our work we focus on understanding the type of defects 
generated by low energy carbon atoms and high dose irradiation and compare to those observed experimentally under different irradiation conditions. 


\section{Methodology}

We have performed molecular dynamics simulations of irradiation of graphene with carbon ions using two different interatomic potentials, Tersoff [14] and Brenner [15]. Both potentials have been fitted to the Universal potential for short distances, required for irradiation simulations. Calculations with the Tersoff potential were done using the MD code LAMMPS [16] and the MDCASK code [17] was used for the Brenner potential calculations.

The simulations consist of a single graphene layer of surfaces between $5 \mathrm{~nm}$ x $5 \mathrm{~nm}$ and $17 \mathrm{~nm}$ x $17 \mathrm{~nm}$. In one direction the borders of the simulation cell are fixed while they are free in the other two directions. For the small simulation cells the system is coupled to a thermal bath that consists of two atomic layers at the border of the simulation cell where the temperature is rescaled every time step. The temperature in these calculations is $10 \mathrm{~K}$. For the larger system size, the simulation cell is first relaxed at $300 \mathrm{~K}$ before the energetic atom is launched towards the graphene layer and no thermal bath is used. Energies of the carbon ion of $100 \mathrm{eV}$ and $200 \mathrm{eV}$ are used. In these calculations the electronic energy loss was neglected since at these low energies this contribution is very small. The coordination of the atoms in the graphene layer is obtained to identify the presence of defects such as vacancies. Figure 1 shows, as an example, the initial conditions for the simulation of a $5 \mathrm{~nm} \times 5 \mathrm{~nm}$ graphene layer, showing the location of the static layers and the free boundaries.

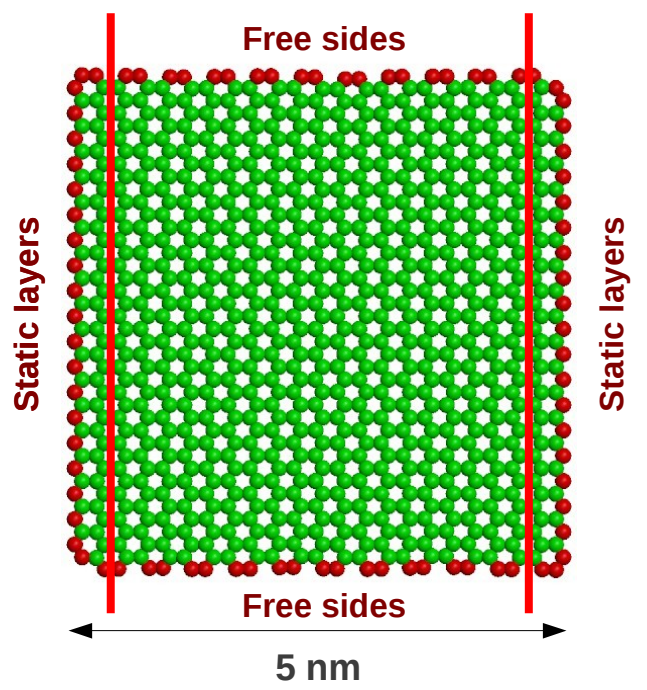

Figure 1: Simulation cell. Single graphene layer of a $5 \mathrm{~nm}$ x $5 \mathrm{~nm}$ surface. Borders along one of the directions are fixed and free on the perpendicular direction.

Ions are introduced in the sample at different rates, between 0.05 picoseconds and 1 picosecond per ion and in areas that go from about $4 \mathrm{~nm}^{2}$ to $54 \mathrm{~nm}^{2}$. Any of the dose rates used in these simulations are orders of magnitude higher than those expected in an ion irradiation experiment. A simulation that reproduces the exact experimental conditions will require times between ions of milliseconds, which is not feasible with molecular dynamics simulations. For such a direct comparison, a combination of molecular dynamics and kinetic Monte Carlo calculations would be needed. However, the purpose of this work is not to directly compare with the experimental results in terms of dose and defect population, but to explore if simulations can reproduce some of the structures observed experimentally and under which conditions. 



\section{Results}

Figure 2 shows the results obtained for system sizes of $5 \mathrm{~nm}$ x $5 \mathrm{~nm}$ with the interatomic potential from Brenner [15] and irradiation with $200 \mathrm{eV}$ carbon atoms. The ions are sent perpendicular towards the surface from random locations in a surface of $4.5 \mathrm{~nm} \times 4.5 \mathrm{~nm}$, that is, the dose per ion is on the order of $5 \times 10^{12} \mathrm{ions} / \mathrm{cm}^{2}$ A total of 80 ions are launched. The time between ions in this case is 1 picosecond. Four snapshots are shown in figure 2 after the irradiation with 10, 20, 30 and 40 ions (figures 2a, 2b, 2c and 2d respectively). Red spheres represent undercoordinated atoms, that is those atoms at the border of the simulation cell and atoms surrounding vacancies and vacancy clusters. Figure 2a shows that after 10 ions a di-atomic chain is formed. Di-atomic chains have been observed experimentally after electron irradiation [6]. Such chain is remarkably strong. After 40 ions, the chain is still present, even though collisions are produced close to this chain and other defects are formed around it. In fact, the vacancy cluster grows significantly without breaking this chain.

\section{Di-atomic chains}

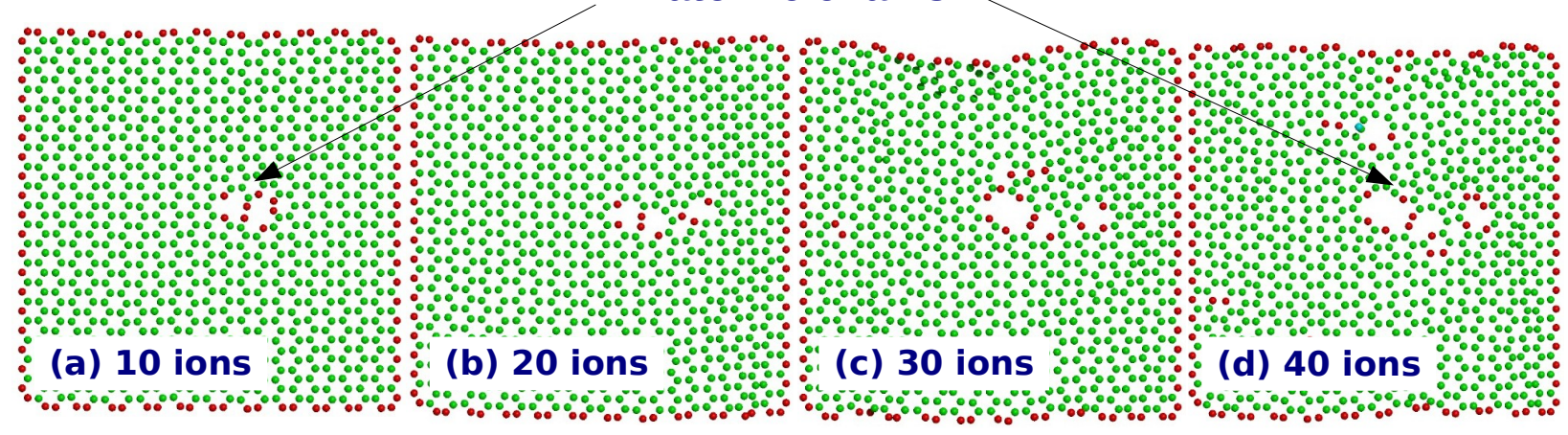

Figure 2: Defects produced in the graphene layer for doses of 10, 20, 30 and 40 carbon ions of $200 \mathrm{eV}$. Note the formation of a very stable di-atomic chain after 10 ions and how it remains even after the implantation of 40 ions.

Higher doses show the formation of more complex structures, also observed experimentally [6]. One of such structures is a parallel chain, close to a large void, as shown in figure 3. The formation of nanopores in suspended graphene has been studied by molecular dynamics simulations by Li et al [12]. In those simulations irradiation was confined to a smaller area (1.5 nm radius) and higher energies were used (500eV to $100 \mathrm{keV})$. Our simulations show that nanopores can be created even at lower energies. Like in the case of reference [12], these pores produced by carbon ions do not have smooth edges and many carbon chains are formed.

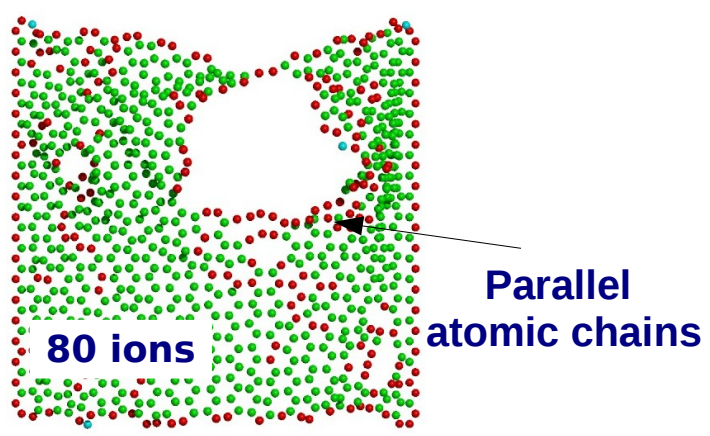

Figure 3: Formation of parallel atomic chains at high implantation dose (80 ions). 
It is important to mention that for this small system size, the implantation of carbon atoms produces a buckling in the graphene layer, as shown in figure 4. In this figure we show a transversal view of the graphene layer after irradiation with 10, 20, 30 and 40 carbon ions of $200 \mathrm{eV}$, corresponding to the same conditions as in figure 2 . The buckling of the graphene layer starts to be apparent after 20 ions, and it is very clear after 40 ions. In fact, deflection of a graphene layer by the implantion with ions has also been studied by molecular dynamics by Terdalkal et al [18]. However, in this case the bucklings seems to be enhanced by the small size of the simulation box and the large number of defects produced at this high dose rate.

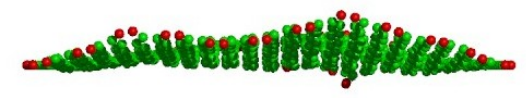

(a) 10 ions

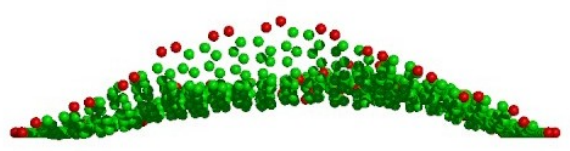

(c) 30 ions

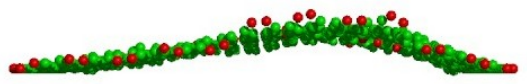

(b) 20 ions

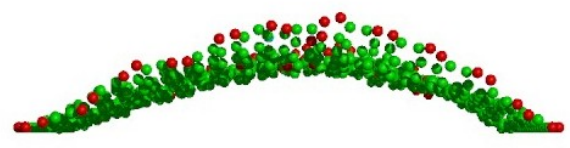

(d) 40 ions

Figure 4: Buckling of the graphene layer due to implantation for different implantation doses.

We have performed simulations with larger system sizes $(17 \mathrm{~nm} \times 17 \mathrm{~nm})$ and $100 \mathrm{eV}$ carbon ions with the interatomic potential of Tersoff [14] coupled to the Universal potential [11]. In this case the ions are also sent perpendicular towards the surface from random locations. Two different areas have been sampled. When the ions are launched in a small area, $3.5 \mathrm{~nm}^{2}$, that is a dose per ion of about $28 \times 10^{12} \mathrm{ions} / \mathrm{cm}^{2}$ (six times higher than the case above, figures 2-4) and at a very high dose rate (one ion every $15 \mathrm{fs}$ ) a nanopore is formed similar to those observed in the simulations by Li et. al [12]. Note that in the work of Li higher energies of the carbon ion are used (500eV to $100 \mathrm{keV})$, similar doses per ion $\left(16 \times 10^{12} \mathrm{ions} / \mathrm{cm}^{2}\right)$ and longer times between ions (1 picosecond between each ion). The nanopore formed after 80 ions in our calculations is shown in figure 5. Interestingly, even though here the ions are distributed in a rectangular region on the surface of the graphene, the nanopore has round and rough edges, like in [12].

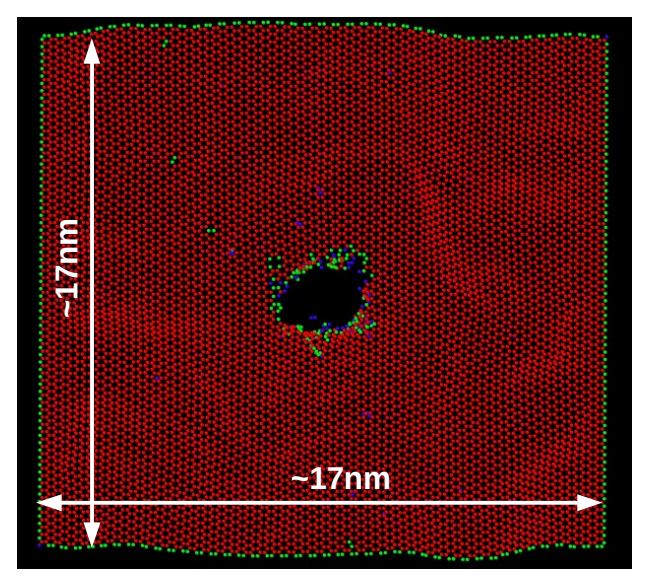

Figure 5: Formation of a nanopore due to irradiation with $100 \mathrm{eV}$ carbon ions at a high dose and dose rate per ion. Image obtained with OVITO [19]. 
These simulations show that the dose per ion as well as the dose rate have a significant impact in the type of structures formed. As mentioned above, increasing the time between incoming ions in the simulation to reach the values expected experimentally is not possible using molecular dynamics. However, the effect of the dose can also be explored by increasing the region where the damage is accumulated such that at low doses, defects are created far away from each other and do not interact, giving them time to relax. In this respect we have performed calculations where the simulation cell is kept constant and equal to the simulation of the nanopore (figure 5), that is a $17 \mathrm{~nm} \mathrm{x}$ $17 \mathrm{~nm}$ cell, but we have extended the region where ions are launched towards the surface. In this case the surface for irradiation was $9 \mathrm{~nm} \times 6 \mathrm{~nm}$ in the centre of the simulation cell, so that the dose per ion is on the order of $2 \times 10^{12}$ ions $/ \mathrm{cm}^{2}$, or 2.5 times lower than in the case of figures 2-4 and 8 times lower than in figure 5. Simulations were performed with time between two consequent ions of 1 picosecond and 50 femtoseconds. Figure 6(a) shows the results for the case after 80 ions with $200 \mathrm{eV}$ carbon ions and 50 femtoseconds between two ions. The result is clearly very different from those presented in figures 2 or 5 . In this case only single vacancies and small vacancy clusters are formed. Correspondingly, the transversal view of the graphene sheet, shown in figure 6(c) shows no significant buckling after irradiation with 80 ions, unlike in the case of figure 4 . This is a consequence of the larger system size and the smaller defects produced. A transversal view of the initial before irradiation and after relaxation at $300 \mathrm{~K}$ is shown in figure 6(b) for comparison with the structure after irradiation with 80 ions (figure 6(c)).
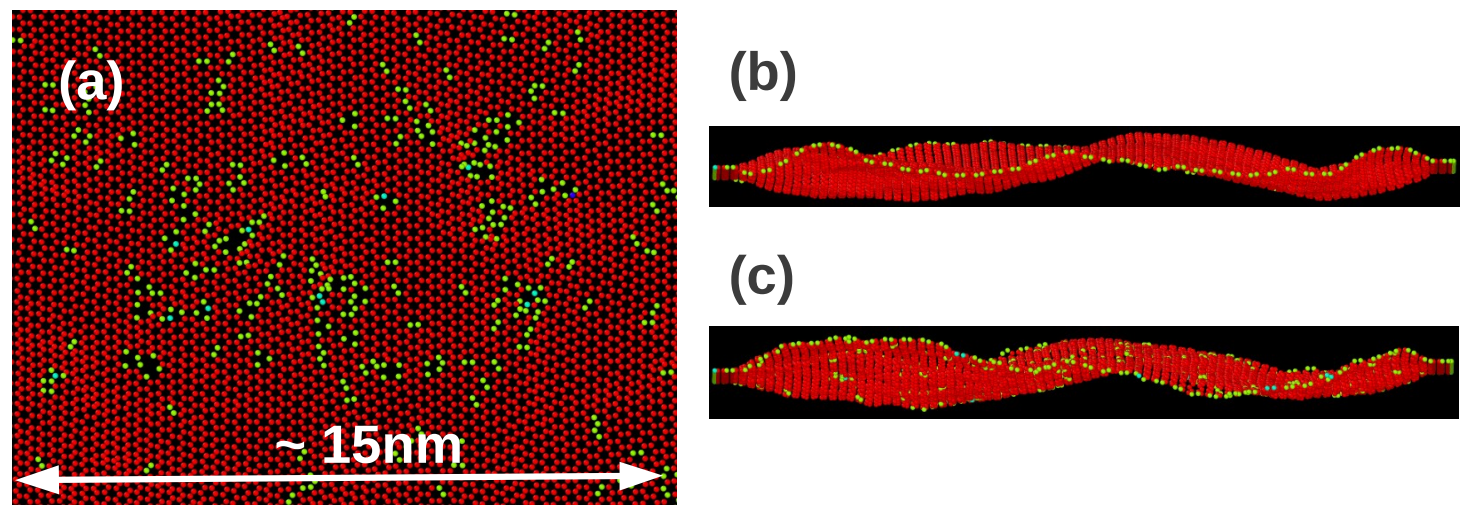

(c)

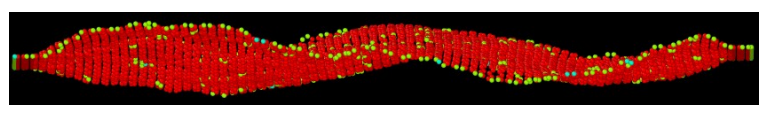

Figure 6: Irradiation with $200 \mathrm{eV}$ carbon ions in a $9 \mathrm{~nm}$ x $6 \mathrm{~nm}$ area and 1 ion every 50 femtoseconds (a) top view after 80 ions (b) lateral view before implantation (c) lateral view after 80 implanted ions. Images obtained with OVITO [19].

\section{Conclusions}

Molecular dynamics simulations of high rate irradiation of graphene with low energy carbon ions (100 eV and $200 \mathrm{eV}$ ) have been performed with different system sizes and interatomic potentials. Simulations reveal the formation of very stable structures such as single chains of carbon atoms and parallel chains of carbon atoms. These structures have been observed experimentally after electron irradiation [6]. For very high dose and dose rates nanopores are formed. These nanopores have been observed in previous simulations by other authors at higher energy. Here we show how these nanopores can be formed even at $100 \mathrm{eV}$ carbon implantation provided the dose rate is very high. Simulations also show that buckling of the graphene layer occurs due to the irradiation for high radiation doses and small systems. 
In general, these calculations show how tunning the different parameters of irradiation, such as dose, dose rate and ion energy, can be used to selectively create different types of defects, from single vacancies to atomic chains of atoms and nanopores in graphene. However, one must keep in mind the high rates used in the calculations compared to experiments. Diffusion processes and relaxation processes can occur in experimental time scales that could prevent the formation of some of these large defect structures. Irradiations at low temperatures could prevent some of these processes to occur and promote the formation of these structures.

\section{Acknowledgements}

We want to thank Professors J. Fernandez-Rossier and C. Untiedt from de UA and Prof. Juan José Palacios from the UAM for introducing us to the field of nanomaterials and graphene in particular. Simulations were performed in the computer cluster and other computer resources of the Dept. of Applied Physics at the UA. This work is supported by the Generalitat Valenciana through grant reference PROMETEO2012/011 and the Spanish government through grant FIS2010-21883.

\section{References}

[1] K. S. Novoselov, V. I. Falḱko, L. Colombo, P. R. Gellert, M. G. Schwab, K. Kim, Nature 490 (2012) 192

[2] M. D. Stoller, S. Park, Y. Zhu, J. An, R. S. Ruoff, Nano Lett 8 (2008) 3498

[3] Y. Wu, Y-m Lin, A. A. Bol, K. A. Jenkins, F. Xia, D. B. Farmer, Y. Zhu, P. Avouris, Nature 472 (2011) 74

[4] Y. Wang, Z. Li, J. Wang, J. Li, Y. Lin, Trends in Biotechnology, 29 (2011) 205

[5] G-D. Lee, C. Z. Wang, B. Yoon, N-M. Hwang, D-Y Kim, K. M. Ho, Phys. Rev. Lett 95 (2005) 205501

[6] C. Jin, H. Lan, L. Peng, K. Suenaga, S. Iijima, Phys. Rev. Lett. 102 (2009) 205501

[7] J. Kotakoski, J. C. Meyer, S. Kurasch, D. Santos-Cottin, U. Kaiser, A. V. Krasheninnikov, Phys. Rev. B 83, (2011) 245420

[8] L. Tapaszto, G. Dobrik, P. Nemes-Incze, G. Vertesy, P. Lambin,

L. P. Biro, Phys. Rev. B 78 (2008) 233407

[9] A. V. Krasheninnikov, K. Nordlund, J. of Appl. Phys. 107 (2010) 071301

[10] K. Kim, V. I. Artyukhov, W. Regan, Y. Liu, M. F. Crommie, B. I. Yakobson, A. Zettl, Nano Lett. 12 (2012) 293

[11] E. P. Bellido, J. M. Seminario, J. of Phys. Chem. C 116 (2012) 4044

[12] W. Li, L. Liang, S. Zhao, S. Zhang, and J. Xue, J. of Applied Phys. 114 (2013) 234304

[13] Y. Asayama, M. Yasuda, K. Tada, H. Kawata, Y. Hirai, J. Vac. Sci. Technol. B 30 (2012) 06FJ02

[14] J. Tersoff, Phys. Rev. Lett. 61 (1988) 2879

[15] D. W. Brenner, Phys. Rev. B 42 (1990) 9458

[16] S. J. Plimpton, Comput. Phys. 117 (1995) 1-19

[17] M. J. Caturla, T. Diz de la Rubia, L. A. Marques, G. Gilmer, Phys. Rev. B 54 (1996) 16683

[18] S. S. Terdalkar, S. Zhang, J. J. Rencis, K. J. Hsia, International Journal of Solids and Structures 45 (2008) 3908-391

[19] A. Stukowski Modelling Simul. Mater. Sci. Eng. 18 (2010), 015012; http://ovito.org/ 